\title{
IDENTIFYING DEGRADATION MECHANISMS IN RF MEMS CAPACITIVE SWITCHES
}

\author{
R.W. Herfst ${ }^{1}$, P.G. Steeneken ${ }^{1}$, and J. Schmitz ${ }^{2}$ \\ ${ }^{1}$ NXP Semiconductors / Corporate I\&T / Research, Eindhoven, The Netherlands. \\ ${ }^{2} \mathrm{MESA}+$ Research Institute, Chair of Semiconductor Components, \\ University of Twente, Enschede, The Netherlands.
}

\begin{abstract}
In this paper we demonstrate how different degradation mechanisms of RF MEMS capacitive switches can be identified by carefully examining changes in key aspects of the measured $\mathrm{C}-\mathrm{V}$ curves. We show that $\mathrm{C}-\mathrm{V}$ curve narrowing can occur either due to mechanical deformation or to laterally inhomogeneous dielectric charging. We also show how these two degradation mechanisms can be distinguished by monitoring the change in the pull-in and pull-out voltages. Our measurements indicate that both degradation mechanisms do indeed occur in practice, depending on the stress conditions.
\end{abstract}

\section{INTRODUCTION}

RF MEMS (Radio Frequency Micro-Electro-Mechanical Systems) capacitive switches have great potential for use in wireless applications because they exhibit good RF characteristics (such as high linearity and low losses) and low power consumption [1]. However, a major challenge for the successful implementation of the switches in commercial products is obtaining a high reliability.

Many papers on the degradation of RF MEMS capacitive switches focussed on dielectric charging [1]-[9]. The main effect of dielectric charging is that it results in a built-in voltage that shifts the entire $\mathrm{C}-\mathrm{V}$ curve [8]-[11]. An additional, but a less documented observation is that the positive and negative pull-in voltage move closer to each other [12], [13]. An explanation for this narrowing of the $\mathrm{C}-\mathrm{V}$ curve was proposed by Rottenberg et al. [14] based on the assumption that the charge is injected inhomogeneously. However, this is not the only explanation for the narrowing effect: we note here that a reduction of the gap due to mechanical degradation of the springs will also reduce the pull-in voltage.

To distinguish which of these two degradation mechanisms causes the narrowing effects when a switch is stressed, we will first show that, given a certain amount of change in the pullin voltage, the amount of change in the pull-out voltage will depend on the degradation mechanism. After this we present measurements which show that both mechanical deformation and inhomogeneous dielectric charging can occur, depending on the type of stress that is applied.

\section{C-V CURVE NARROWING THEORY}

In Fig. 1 a SEM picture of a MEMS switch such as used in this article is shown. The switch consists of two electrodes, a dielectric layer, and an air gap. The top electrode is suspended by springs. In our case the electrode material is an aluminium alloy, and the dielectric is made of silicon nitride.

In a simplified model for a capacitive MEMS switch it is

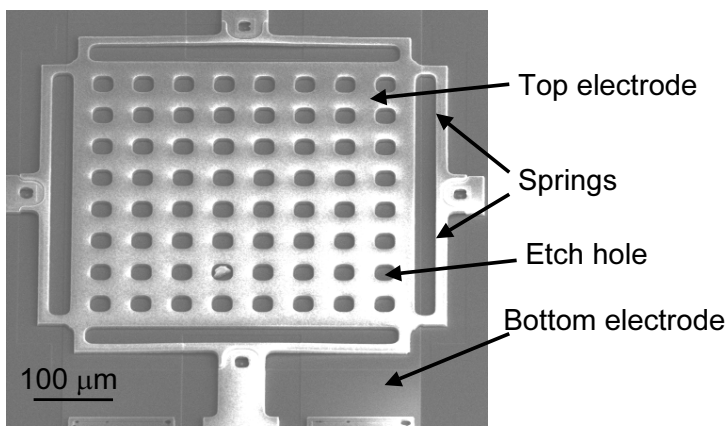

Fig. 1. SEM picture of an RF MEMS capacitive switch. Electrodes are made of an aluminum alloy, the dielectric consists of $\mathrm{Si}_{\mathrm{x}} \mathrm{N}_{\mathrm{y}}$.

viewed as two rigid parallel plates with area $A$, with the top plate suspended by a linear spring with spring constant $k$. For such a system [1] the electrostatic force is equal to

$$
F_{E}=-\frac{\epsilon_{0} A}{2} \frac{V^{2}}{\left(d / \epsilon_{r}+g\right)^{2}},
$$

where $\epsilon_{0}$ is the dielectric constat, $d$ the thickness of the dielectric, $\epsilon_{r}$ the relative dielectric permittivity, and $g$ the gap between the top of the dielectric and the bottom of the top electrode. For the spring force we have

$$
F_{\text {spring }}=-k\left(g-g_{0}\right),
$$

where $g_{0}$ is the gap when no electrostatic force is applied. When a voltage is applied across the two electrodes, the electrode will be pulled downward until $F_{E}$ is in equilibrium with $F_{\text {spring. }}$. Above a certain voltage, called the pull-in voltage $V_{\text {pi }}$, the balance between $F_{E}$ and $F_{\text {spring }}$ becomes unstable and the switch closes, which is marked by a sudden increase in the capacitance. The solution for this is:

$$
V_{\mathrm{pi}}^{ \pm}= \pm \sqrt{\frac{8 k\left(d / \epsilon_{r}+g_{0}\right)^{3}}{27 \epsilon_{0} A}}
$$

where $V_{\mathrm{pi}}^{+}$is the positive pull-in voltage and $V_{\mathrm{pi}}^{-}$the negative pull-in voltage. Since the distance between the two electrodes is relatively small in the closed state, the electrostatic force is larger than the restoring force in the closed state (equilibrium is maintained by contact forces), so that when the voltage is decreased again, the switch will not open at $V=V_{\mathrm{pi}}$ but at a lower voltage, the pull-out voltage $V_{\mathrm{po}}$ :

$$
V_{\text {po }}^{ \pm}= \pm \sqrt{\frac{2 k g_{0}\left(d / \epsilon_{r}\right)^{2}}{\epsilon_{0} A}} .
$$

An example of the capacitance as function of voltage, with $V_{\mathrm{pi}}^{ \pm}$and $V_{\mathrm{po}}^{ \pm}$is shown in Fig. 2. 


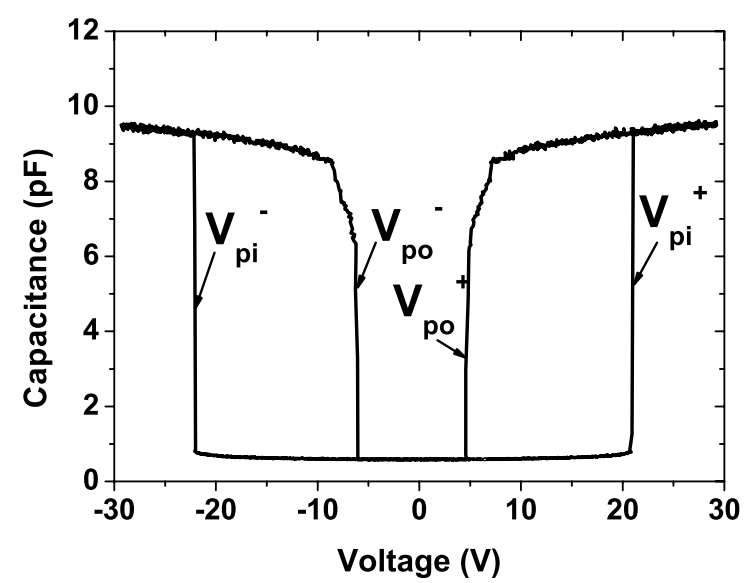

Fig. 2. C-V curve example of the used MEMS switches with pull-in and pull-out voltages.

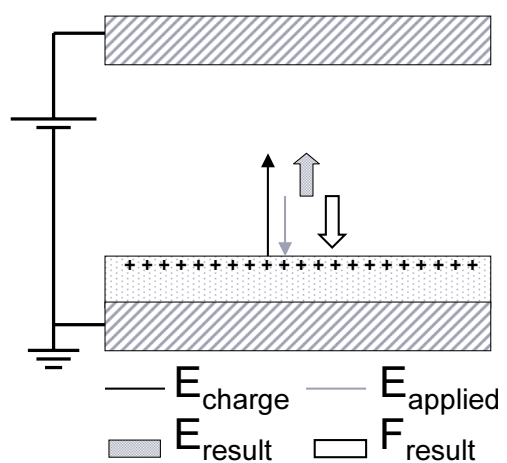

Fig. 3. Schematic representation of how trapped charge change the electric field and electrostatic force in a capacitive switch.

MEMS reliability studies have shown [1]-[9] that due to the high electric fields charge can be injected in the dielectric. The trapped charges change the electric field (Fig. 3) and lead to a built-in voltage $V_{\text {shift }}$ which shifts the C-V curve [8]-[11]. This $V_{\text {shift }}$ is proportional to the amount of charge, and to the distance between the trapped charge and the bottom electrode. However, this does not explain the narrowing observed in [6] and [12]. Three causes can account for this narrowing effect:

1 A laterally inhomogeneous distribution of the trapped charge [14].

2 A reduced open state gap $g_{0}$ due to mechanical deformation.

3 A reduction of the spring constant $k$.

Of these three causes, the third one is not very likely, as the spring constant is only a function of the shape of the springs and its material properties, which both do not change significantly during stress tests. The first two causes are discussed in more detail below.

\section{A. Laterally inhomogeneous dielectric charging}

When charge is injected inhomogeneously, the expression for the electrostatic force changes from Eq. 1 to [14]:

$$
\begin{aligned}
F_{E} & =-\frac{\epsilon_{0} A}{2} \int_{\text {Area }} \frac{\left(V-V_{\text {shift }}(x, y)\right)^{2}}{\left(d / \epsilon_{r}+g\right)^{2}} \mathrm{~d} A \\
& =-\frac{\epsilon_{0} A}{2} \frac{\left(V-\bar{V}_{\text {shift }}\right)^{2}+\sigma_{V_{\text {shift }}^{2}}^{2}}{\left(d / \epsilon_{r}+g\right)^{2}}
\end{aligned}
$$

where $V_{\text {shift }}(x, y)$ is the position dependent built-in voltage, $\bar{V}_{\text {shift }}$ the average of $V_{\text {shift }}(x, y)$, and $\sigma_{V_{\text {shift }}}$ standard deviation of $V_{\text {shift }}(x, y)$. From the equation we see that by applying a voltage we can only compensate for the contribution to $F_{E}$ of $\bar{V}_{\text {shift }}$, and always have the additional force due to $\sigma_{V_{\text {shift }}}$. When comparing this with Eq. 1 we see that the $g$ dependence has not changed, and if the open state gap has not changed, the force balance remains essentially the same. In the inhomogeneously charged situation pull-in will occur when the value of the nominator is equal to the value it has for the uncharged situation at pull-in:

$$
\begin{aligned}
& \left(V_{\mathrm{pi}}-\bar{V}_{\text {shift }}\right)^{2}+\sigma_{V_{\text {shift }}}^{2}=V_{\mathrm{pi},(t=0)}^{2} \Longrightarrow \\
& V_{\mathrm{pi}}^{ \pm}=\bar{V}_{\text {shift }}+V_{\mathrm{pi},(t=0)}^{ \pm} \sqrt{1-\sigma_{V_{\text {shift }}}^{2} / V_{\mathrm{pi},(t=0)}^{2}},
\end{aligned}
$$

where $V_{\mathrm{pi},(t=0)}^{ \pm}$is the pull-in voltage of the unstressed device. A similar argument can also be made for the pull-out voltage, where we find that

$$
\begin{aligned}
& \left(V_{\mathrm{po}}-\bar{V}_{\text {shift }}\right)^{2}+\sigma_{V_{\text {shift }}}^{2}=V_{\mathrm{po},(t=0)}^{2} \Longrightarrow \\
& V_{\mathrm{po}}^{ \pm}=\bar{V}_{\text {shift }}+V_{\mathrm{po},(t=0)}^{ \pm} \sqrt{1-\sigma_{V_{\text {shift }}}^{2} / V_{\mathrm{po},(t=0)}^{2}},
\end{aligned}
$$

Here $V_{\mathrm{po},(t=0)}^{ \pm}$is the pull-out voltage of the unstressed device. Since by definition $V_{\text {po }}$ is smaller than $V_{\mathrm{pi}}$, inhomogeneous charging will cause a larger change in $V_{\mathrm{po}}$ than in $V_{\mathrm{pi}}$. Also, Eq. 7 shows that when $\sigma_{V_{\text {shift }}}$ is equal to $V_{\mathrm{po},(t=0)}$, the switch will not open anymore.

\section{B. Mechanical degradation of the springs}

When the open state gap is reduced by mechanical degradation of the springs, but no inhomogeneous charge is injected, the change in $V_{\mathrm{pi}}$ can be found from the 1-D solutions for pull-in:

$$
\begin{aligned}
& V_{\mathrm{pi}}^{ \pm}= \pm \sqrt{\frac{8 k\left(d / \epsilon_{r}+g_{0}\right)^{3}}{27 \epsilon_{0} A} \Longrightarrow} \\
& V_{\mathrm{pi}}^{ \pm}=V_{\mathrm{pi},(t=0)}^{ \pm} \sqrt{\frac{\left(d / \epsilon_{r}+g_{0}\right)^{3}}{\left(d / \epsilon_{r}+g_{0,(t=0)}\right)^{3}}},
\end{aligned}
$$

where $g_{0,(t=0)}$ is $g_{0}$ of the undeformed device. The change in $V_{\text {po }}$ can be found from the 1-D solution for pull-out:

$$
\begin{aligned}
& V_{\mathrm{po}}^{ \pm}= \pm \sqrt{\frac{2 k g_{0}\left(d / \epsilon_{r}\right)^{2}}{\epsilon_{0} A}} \Longrightarrow \\
& V_{\mathrm{po}}^{ \pm}=V_{\mathrm{po},(t=0)}^{ \pm} \sqrt{\frac{g_{0}}{g_{0,(t=0)}}} .
\end{aligned}
$$

From these two equations we can see that a change in $g_{0}$ will have a greater effect on $V_{\text {pi }}$ then on $V_{\text {po }}$. 


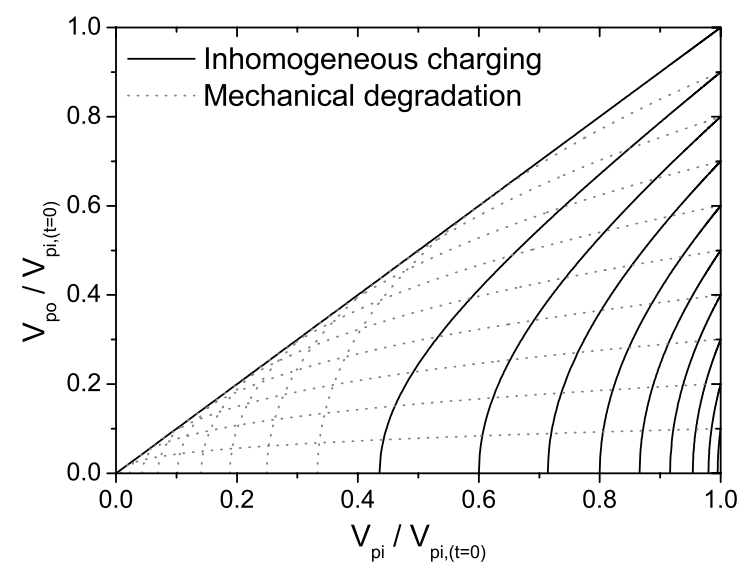

Fig. 4. $V_{\mathrm{po}}$ (normalized to $V_{\mathrm{pi}}$ at $t=0$ ) as function of normalized $V_{\mathrm{pi}}$ when a switch degrades. When $V_{\text {po }}$ becomes zero, a device will fail.

When $V_{\mathrm{po}}$ (normalized to $V_{\mathrm{pi},(t=0)}$ ) is plotted as function of the normalized $V_{\mathrm{pi}}$ for increasing inhomogeneous charge $\sigma_{V_{\text {shift }}}$ and decreasing gap $g_{0}$, the lines as shown in Fig. 4 are obtained, which clearly indicate the different relation between $V_{\mathrm{pi}}$ and $V_{\mathrm{po}}$ for the two degradation mechanisms. Devices will fail when $V_{\text {po }}$ becomes zero. We see that pristine devices with a low pull-out voltage (thin dielectric) can withstand a lot of mechanical degradation but only little inhomogeneous charging, whereas devices starting with a relatively high pullout voltage can withstand more inhomogeneous charging, but less mechanical degradation.

\section{Combined effect}

If $\mathrm{C}-\mathrm{V}$ curve narrowing is due to a combination of mechanical degradation and inhomogeneous charging, Eq. 6, 7, 8 , and 9 can be combined to the more general equations

$$
V_{\mathrm{pi}}^{ \pm}=\bar{V}_{\mathrm{shift}}+V_{\mathrm{pi},(t=0)}^{ \pm} \sqrt{\frac{\left(d / \epsilon_{r}+g_{0}\right)^{3}}{\left(d / \epsilon_{r}+g_{0,(t=0)}\right)^{3}}-\frac{\sigma_{V_{\text {shift }}}^{2}}{V_{\mathrm{pi},(t=0)}^{2}}},
$$

and

$$
V_{\mathrm{po}}^{ \pm}=\bar{V}_{\mathrm{shift}}+V_{\mathrm{po},(t=0)}^{ \pm} \sqrt{\frac{g_{0}}{g_{0,(t=0)}}-\frac{\sigma_{V_{\text {shift }}}^{2}}{V_{\mathrm{po},(t=0)}^{2}}} .
$$

Although $\bar{V}_{\text {shift }}$ can easily be eliminated by measuring both the positive and negative pull-in and pull-out voltages, solving for $\sigma_{V_{\text {shift }}}$ and $g_{0}$ yields rather complicated solutions and is best handled by symbolic maths computer programs such as Mathematica or Maple.

\section{MEASUREMENTS}

To establish which mechanism is the most important for $\mathrm{C}-\mathrm{V}$ curve narrowing of our switches, we measured the $\mathrm{C}-\mathrm{V}$ curve as function of the applied stress. The $\mathrm{C}-\mathrm{V}$ curves were obtained by measuring the reflection coefficient of a $890 \mathrm{MHz}$ RF signal while applying a triangle wave bias voltage. This system is described in [13] and ensures that C-V curves are measured quickly enough ( $1 \mathrm{C}-\mathrm{V}$ curve in $400 \mathrm{~ms}$ ) to not have significant charging effects during the measurement of the $\mathrm{C}-\mathrm{V}$
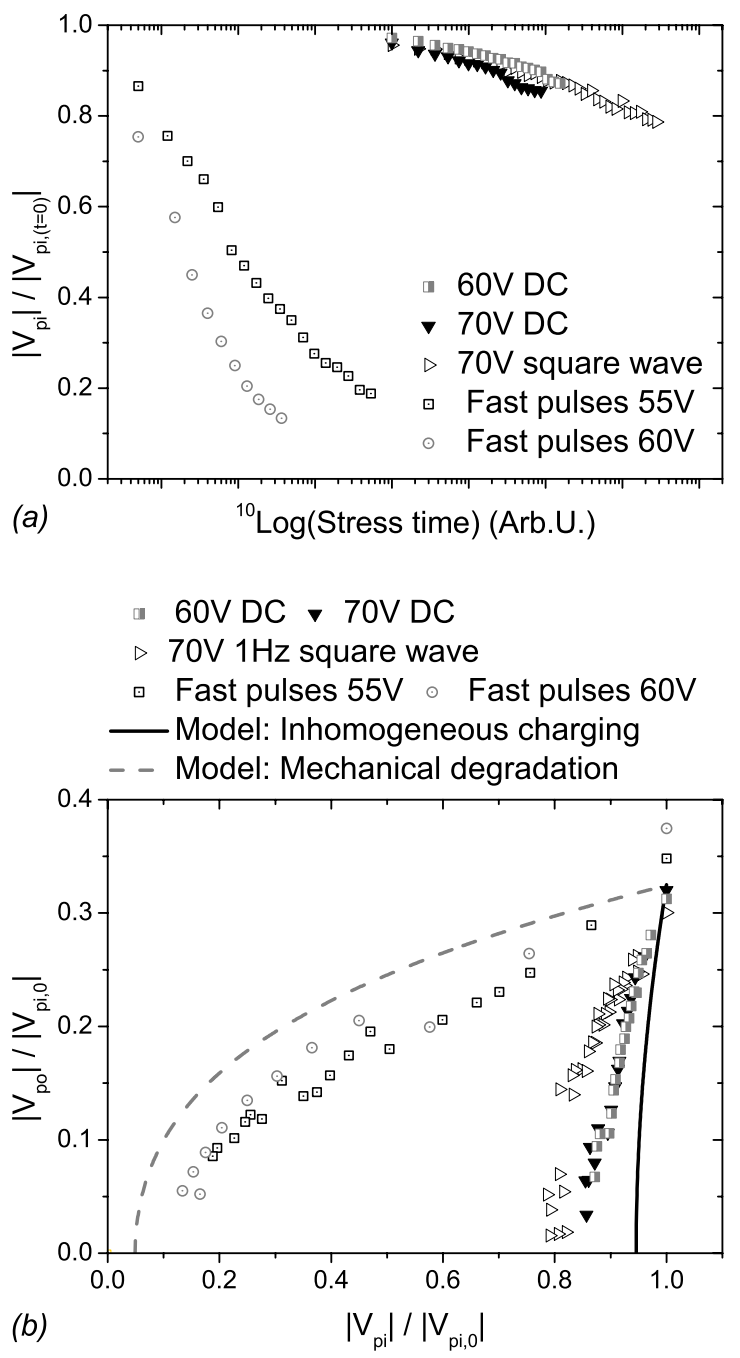

Fig. 5. Measured changes in the width of the $C$-V curve. (a) $\left|V_{\mathrm{pi}}\right| /\left|V_{\mathrm{pi},(t=0)}\right|$ as function of stress time. $(b)\left|V_{\mathrm{po}}\right| /\left|V_{\mathrm{pi},(t=0)}\right|$ as function of $\left|V_{\mathrm{pi}}\right| /\left|V_{\mathrm{pi},(t=0)}\right|$. We define $\left|V_{\mathrm{pi}}\right|$ as $\left(V_{\mathrm{pi}}^{+}-\right.$ $\left.V_{\text {pi }}^{-}\right) / 2$.

curve. From these C-V curves $V_{\mathrm{pi}}^{ \pm}$and $V_{\text {po }}^{ \pm}$were extracted. We used three different stress patterns: a DC bias voltage, a $1 \mathrm{~Hz}$ symmetric square wave (both actuated in a way that minimizes the impact velocity), and $200 \mathrm{~Hz}$ fast pulses (actuated with a high impact velocity). The difference in impact velocity was realized by changing the RC-time of the bias-T that was used to add the bias voltage to the RF signal used for measuring the capacitance. When the RC-time is much longer than the switching time, the switch closes while the voltage across the switch is equal to the pull-in voltage, whereas when the RCtime is much shorter than the switching time, the switch closes while the voltage across the switch is equal to the bias voltage, which can be significantly higher than the pull-in voltage, thus causing a higher impact velocity.

In Fig. 5a the normalized $V_{\mathrm{pi}}$ is plotted as function of stress time. We see that devices actuated with the fast pulses degrade much more rapidly than devices stressed with DC and square wave actuation. The reason for this large difference 


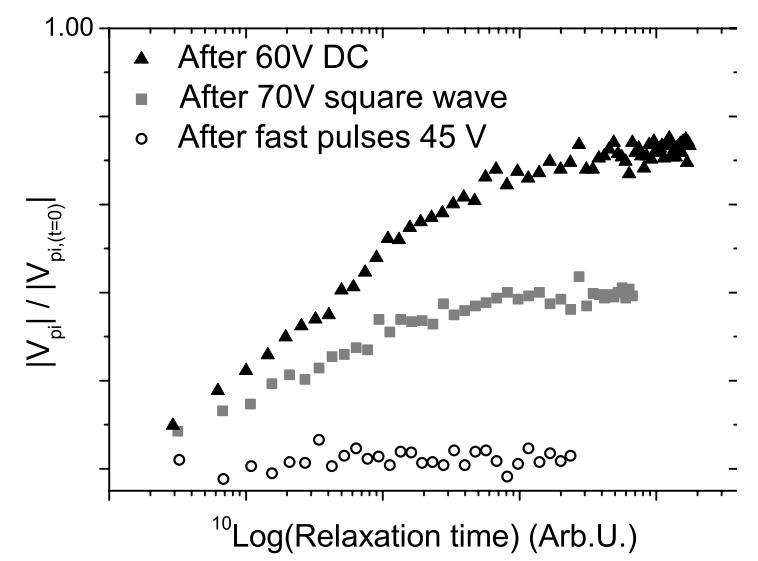

Fig. 6. Relaxation of the width of the $C$-V curve after narrowing.

becomes clear in Fig. 5b, where $V_{\text {po }}$ is plotted as function of $V_{\mathrm{pi}}$, both normalized to $V_{\mathrm{pi},(t=0)}$. Here we see that in the DC bias voltage case the points lie close to the inhomogeneous charging line, indicating that the narrowing is primarily caused by inhomogeneous charging. In the case of the fast pulses, results are much closer to the mechanical degradation line, indicating that narrowing is caused by mechanical degradation of the springs. The deviations from the theoretical lines could be caused by combined effects of inhomogeneous charging and mechanical degradation (section 2-C). It might also be caused by the way the switch is modelled: since we regard the top electrode as a rigid plate, higher order effects on $V_{\mathrm{pi}}$ and $V_{\mathrm{po}}$ such as roll-of are not taken into account. The square wave actuation results are close to the inhomogeneous charging line, but not as close as the DC bias voltage results, which suggests that their $\mathrm{C}-\mathrm{V}$ curve narrowing is due to the combined effect of mechanical degradation and inhomogeneous charging.

The differences in degradation mechanisms can also be observed if stressed devices are allowed to relax (Fig 6): the narrowing due to the DC bias voltage is almost completely reversible, which indicates that the narrowing was not caused by plastic deformation of the springs, since that would be permanent. The damage due to the fast actuation pulses is permanent, strongly suggesting plastic deformation has occurred. Devices stressed with the square wave actuation are between the other two, and show only partial recovery.

From the measurements we can conclude that when the impact velocity increases, the main failure mode shifts from inhomogeneous charging to mechanical degradation. Lifetime is then no longer limited by the amount of time the switch is in the closed position, but by the amount of stress cycles.

\section{CONCLUSIONS}

To conclude, we provided a framework with which the relation between degradation mechanism, stress conditions, and actuation scheme can be identified. We used it to demonstrate for the first time that, depending on the stress conditions, degradation of RF MEMS capacitive switches does not only occur due to (inhomogeneous) dielectric charging, but can also be attributed to plastic deformations of the springs. Lifetime is then no longer limited by the amount of time the switch is in the closed position, but by the amount of stress cycles.

\section{ACKNOWLEDGEMENTS}

We thank our colleagues at NXP Semiconductors Nijmegen and Eindhoven for providing samples of the RF MEMS capacitive switches and fruitful discussions on MEMS reliability.

\section{REFERENCES}

[1] Gabriel M. Rebeiz, RF MEMS - Theory, Design, and Technology, John Wiley \& Sons, Inc, 2003.

[2] C. Goldsmith, J. Ehmke, A. Malczewski, B. Pillans, S. Eshehnan, Z. Yao, J. Brank and M. Eberly, Lifetime characterization of capacitive RF MEMS switches, 2001 IEEE MTT-S Int. Microwave Symp. Digest, vol. 1, pp. 227-230, May 2001.

[3] S. Melle, F. Flourens, D. Dubuc, K. Grenier, P. Pons, F. Pressecq, J. Kuchenbecker, J.L. Muraro, L. Bary and R. Plana, Reliability overview of RF MEMS devices and circuits, $33^{\mathrm{rd}}$ European Microwave Conference, vol. 1, pp. 37-40, October 2003.

[4] R.W. Herfst, H.G.A. Huizing, P.G. Steeneken, and J. Schmitz, Characterization of dielectric charging in RF MEMS capacitive switches, 2006 IEEE International Conference on Microelectronic Test Structures, 133-136, 6-9 March 2006.

[5] Xiaobin Yuan, James C.M. Hwang, David Forehand, and Charles. L. Goldsmith, Modeling and characterization of DielectricCharging Effects in RF MEMS Capacitive Switches, 2005 IEEE MTT-S Int. Microwave Symp. Digest.

[6] R.W. Herfst, P.G. Steeneken, and J. Schmitz, Time and voltage dependence of dielectric charging in RF MEMS capacitive switches, $45^{\text {th }}$ Annual IEEE International Reliability Physics Symposium, 417-421, 15-19 April 2007.

[7] Giorgos Papaioannou, Michael-Nicolas Exarchos, Vasilios Theonas, Guoan Wang, Student Member, IEEE, and John Papapolymerou, Senior Member, IEEE, Temperature Study of the Dielectric Polarization Effects of Capacitive RF MEMS Switches, IEEE Transactions on Microwave Theory and Techniques, vol. 53 , no. 11,3467 , november 2005.

[8] W. Merlijn van Spengen, Robert Puers, Robert Mertens, and Ingrid De Wolf, A comprehensive model to predict the charging and reliability of capacitive RF MEMS switches, J. Micromech. Microeng. vol. 14, 514-521, 2004.

[9] I. Wibbeler, G. Heifer and M. Hietschold, Parasitic charging of dielectric surfaces in capacitive microelectromechanical systems (MEMS), Sensors and Actuators A: Physical, pp. 74-80, November 1998.

[10] J.R. Reid, Dielectric charging effects on capacitive MEMS actuators, 2002 IEEE MTT-S Int. Microwave Symp. Digest, RF MEMS workshop, June 2002.

[11] S.S. McClure, L. D. Edmonds, R. Mihailovich, A.H. Johnston, P. Alonzo, J. DeNatale, J. Lehman, and C. Yui, Radiation effects in micro-electromechanical systems (MEMS): RF Relays, IEEE Trans. On Nuclear Science, vol. 49, no. 6, December 2002.

[12] J.R. Reid, and R.T. Webster, Measurements of charging in capacitive microelectromechanical switches, ELECTRONICS LETTERS, vol. 38, no. 24, $21^{\text {st }}$ November 2002.

[13] R.W. Herfst, P.G. Steeneken, J. Schmitz, Time and voltage dependence of dielectric charging in RF MEMS capacitive switches, Proceedings $45^{\text {th }}$ Annual IEEE International Reliability Physics Symposium, pp. 417-421, 2007.

[14] X. Rottenberg, B. Nauwelaers, W. De Raedt, and H.A.C. Tilmans, Distributed dielectric charging and its impact on $R F$ MEMS devices, $34^{\text {th }}$ European Microwave Conference - Amsterdam, 2004. 